\title{
TA'LIMIY KORPUSLAR UCHUN GIPONIMIK, GRADUONIMIK VA PARTONIMIK MUNOSABATLAR BILDIRUVCHI BIRLIKLAR BAZASINI SHAKLLANTIRISH
}

\author{
Xoliyorov O'ral Mengliyevich \\ Termiz davlat universiteti katta o'qituvchisi \\ xoliyorov@tersu.uz
}

Annotatsiya. Maqolada leksik-semantik munosabatlar bildiruvchi birliklar bo'yicha olib borilgan tadqiqotlar, manbalar tahlil etilgan. Ta'limiy semantik ma'lumotlar bazasini, ya'ni giponimik, graduonimik, partonimik munosabatlar bildiruvchi birliklar bazasini shakllantirish yuzasidan takliflar berilgan.

Kalit so'zlar: ta'limiy korpus, teg, teglash, giponim, giperonim, xolo/meronim, graduonimiya, jins-tur, butun-bo'lak.

Ta'limiy korpus milliy tilning lug'at boyligi, ularning izohi, ma'nolari, nutqiy qo'llanishini o'rganish uchun qulay interfeysga ega bo'lgan, til o'rgatishga ixtisoslashgan elektron lingvistik, didaktik vositadir. $\mathrm{O}^{`} z$ zek tili ta'limiy korpusida so'z qidiruvi, so'z ma'nosi(lari) ifodalangan oynani tuzish, izohlarni o'rinlartirish, ularning tushunarli bo'lishiga erishish muammolari yechilishi zarur. Til korpusi nafaqat lingvodidaktik, balki lingvistik tadqiq sohalarining ham ish quroli. Korpusning so' $\mathrm{z}$ o'rganishda katta axborot bazasi vazifasini bajarishi allaqachon isbotini topgan.

V.Zaxarov, Y.Bogdanova [Zaxarov, 2011: 16]ning fikriga ko'ra, korpus so'zning barcha ma'nolari, leksik-semantik munosabatga kirishadigan so'zlari bilan yonma-yon izohlashiga ko'ra izohli lug'at, tezaurusdan farq qiladi. Izohli lug'atda faqat so'z ma'nosi izohlansa, tezaurus so'zning nisbatan ko'proq qirralarini ochishga xizmat qiladi. Ammo bu axborot manbalari qidiruv imkoniyatining cheklanganligi bilan korpusdan farq qiladi. Millionlab matn(so'z)larni qamrab olgan til korpuslari so'zni izohlash, uni tildagi munosabatlari bilan birga ko'rsata olish imkoniyatining mavjudligi bilan yuqorida sanalgan axborot manbalaridan ajralib turadi. Shunday ekan o'zbek tili korpusida ham barcha leksik-semantik munosabatga kirishadigan birliklarni aks ettirish, semantik teglash asosiy vazifalardan biri.

O'zbek korpus lingvistikasida milliy korpus uchun birliklarni semantik teglash, ma'nolar haqida teg (izoh) biriktirish, semantik ma'lumotlar bazasini 
shakllantirish bo'yicha bir necha tadqiqotlar amalga oshirilgan [Xamroyeva, 2018; Eshmo'minov, 2019; Axmedova, 2020]. Sh.Xamroyeva semantik teglarni ishlab chiqishning umumiy tamoyillari, A.Eshmo'minov milliy korpusning sinonim so'zlar bazasini shakllantirish, sinonim so'zlar izohining korpusda berilishi, bazadan joy olishi muammolarini tadqiq etgan bo'lsa, D.Axmedova o'zbek tili milliy korpusida so 'zlararo leksik-semantik munosabatning ifodalanishi masalasini o'rgangan. Tadqiqotda so'zning ma'nosini izohlash yoki so'z ma'nolariga yondashuvning ikki tamoyili farqlangan, har bir tamoyildan foydalanish o'rni ko"rsatib berilgan. D.Axmedova leksikaga yondashuvning "tushunchadan so"zga", "so'zdan tushunchaga" kabi ikki turini ko"rsatadi. Uning fikricha, korpusda leksikaga semantik maydon nazariyasi asosidagi yondashuv bilan tushuncha orqali so'zga borilsa, so'zlararo leksik-semantik munosabat orqali so'zdan tushuncha izohiga borish mumkin [Axmedova, 2020].

Tadqiqotchi to'g'ri ta'kidlaganidek, korpus so'zning qo'llanish kontekstiga havola qilishi, so'zning birikuvchanlik imkoniyatini namoyon qilishi uning semantik xususiyat(lar)ini ko'rsatmaydi. Foydalanuvchi qidirayotgan so $^{\text {' } z \text { haqida }}$ batafsil ma'lumotni bir joydan topishiga erishilsa, ma'lum qulaylik kelib chiqadi. D.Axmedova semantik teglashning "so"zdan tushunchaga" tamoyilini ishlab chiqar ekan, so'z ma'nolari izohi, leksik-semantik munosabatga kirishuvchi leksemalari: sinonim, antonim, giponim/giperonim, graduonimi, xolo/meronimi haqidagi izohlarni teg sifatida biriktirish yo'llarini ko'rsatadi. Semantik teglar tizimiga so'zshakl, lemma, izohli lug'atdagidek ma'nolari, mavjud sinonim, graduonim, giponim/giperonim, xolo/meronim, antonimlari haqidagi izoh kiritish lozimligini ta'kidlaydi. Ammo bu xususiyatlarni ochish uchun izohni qay tartibda keltirish, bazani yaratish masalasini ochiq qoldiradi.

O‘zbek tili korpusini tuzishda sinonim, antonim birliklar bazasi uchun yetarli tadqiqotlar, manbalar, lug'atlar mavjud. Lekin giponim/giperonim, xolo/meronimlar bo'yicha tadqiqotlar mavjud bo'lsa-da, lug'atlar yaratilmagan.

Jahon tilshunosligida J.Layonz so'zlararo ma'no munosabatlari doirasida yangi - tur-jins munosabatini ifodalovchi giponimiya hamda giponimik munosabatlar bilan bog'langan so'zlar sirasi, giponimik lug'aviy ma'no guruh(LMG)lar, giponim, giperonim kabi tushuncha va atamalarini olib kirdi [Layonz, 1978]. O`zbek tilshunosligida giponimiya hodisasining o'rganilishi R.Safarova tadqiqotlari bilan bog'liq [Safarova, 1987]. Dunyodagi boshqa tillar kabi o'zbek tilining ham jins-tur munosabatini ifodalash asosida bog'langan lug'aviy birliklari mavjud: ularning so ${ }^{6} \mathrm{z}$ birikmasi vositasida ifodalash imkoniyati 
cheklanmagan. Jins-tur munosabatini ifodalashning leksik imkoniyatlari o'zbek tilida ma'lum lug'aviy birliklar yordamida ifodalanadi. Tilning leksik-semantik tizimida jins belgisining nomini hamda tur belgisining nomini ifodalab kelgan mustaqil lug'aviy birlik mazkur til qurilishidan mustaqil birlik sifatida o'rin oladi. Bunday lug'aviy birlik alohida o'zak morfemaga ega, u tilning mustaqil birligi. Shuning uchun ham bunday lug'aviy birliklarning miqdori har bir tilda qat'iy ravishda belgilab qo'yilgan [Safarova, 1987].

So'zning giponimik qatori giperonim hamda giponimdan iborat. Giperonim - jins belgisini bildirgan predmetning nomini ifodalovchi bir necha ma'noni semantik jihatdan umumlashtiruvchi mikrotizimning markaziy so'zi, dominantasi. Giponim - ma'lum jins turining nomini ifodalovchi hamda o'zining semantik tarkibida jins ma'nosini ifodalovchi so'zni biriktirib kelgan, semantik jihatdan giperonimga nisbatan boy bo'lgan lug'aviy birlik [Safarova, 1987]. O'zbek tilida giperonim va giponim orasida semantik, logik-semantik aloqa mavjud. Bunday lingvistik bazani shakllantirish uchun material vazifasini bajaradigan lug'at mavjud bo'Imasa-da, quyidagicha shakllantirish mumkin:

o'zbek tilining umumiy leksik bazasi olinadi;

umumiy baza bir ustunga qo'yilgandan keyin yonidan yana bir ustun qo'shiladi;

umumiy leksik ba'za giponim sifati belgilanib, yonidagi ustunga giperonimi kiritiladi;

dasturchi yonma yon ustunlardagi birliklarni bir-biriga giperhavola orqali biriktiradi.

Natija barcha so'zlar bir-biriga giponim-giperonim munosabatda bog'lanib qoladi.

\begin{tabular}{|l|l|}
\hline \multicolumn{1}{|c|}{ giponim } & \\
\hline jo 'ja & tovuq \\
\hline terak & daraxt \\
\hline xo'roz & tovuq \\
\hline tol & daraxt \\
\hline gilos & daraxt \\
\hline
\end{tabular}

Ba'zan bir leksemaning turli nuqtai nazardan yoki ma'nolari kengligidan bir nechta giperonimlarga bog'lanishi mumkin. Masalan, olma, gilos, shaftoli, o'rik kabi leksemalar daraxt giperonimi bilan bog'lanishi qatorida meva geperonimi bilan ham birikadi. Bunday hollarda ustunlar yoniga yana ustunlar qo'shib ketaverish mumkin va giperonim1, giperonim2 nomlari bilan nomlasa bo'ladi.

O'zbek tili graduonimik munosabatdagi birliklar bazasi haqida. O'zbek tili lug'at boyligini lug'aviy ma'no guruhlarga ajratish so'zlararo ma'noviy 
munosabatni o'rganish bilan chambarchas bog'liq. Lug'aviy birliklar o'zaro ma'noviy munosabatga ko'ra ma'lum bir darajalanish qatorini hosil etishi tilshunosliqda qadimdan ma'lum. Buning eng yorqin namunasi "o'rta" so'zini o'z ichiga olgan so'zlar qatoridir: katta - o'rta - kichik; yosh - o'rta - qari; uzoq o'rta-yaqin; baland -o'rta - past; uzun - o'rta - qisqa [Orifjonova, 1996].

Sh.Orifjonova graduonimik munosabatni ajratishning lisoniy omili sifatida ma'noviy hamda so'zlararo paradigmatik munosabat omilini farqlaydi. Graduonimik qatorlarni ajratishda ma'noviy omilning mohiyati shundaki, bir qator so'zlardagi atash-nomlash semalari tarkibida miqdorga - ma'lum bir belgining ozko'pligi, turli xil darajalarga ishora mavjud [Orifjonova, 1996]. Bu so'zlarning lisoniy ma'no izohlarida atash, nomlash semalari tarkibida miqdoriy ko'rsatkichlarga, xususiyatlarga ishora qiluvchi semalar, tarkibiy qismlar yaqqol ko'zga tashlanib turibdi.

O'zbek tili ta'limiy korpusi darajalanish qatori ma'lumotlar bazasini shakllantirish uchun, kam bo'lsa-da, materialning borligi quvonarli hol. $\mathrm{Bu}$ ma'lumotlar bazasini shakllantirishda monografik tadqiqot natijalari [Orifjonova, 1996], o'quv darajalanish lug'ati materiallaridan foydalaniladi. O'zbek tilida darajalanish qatori aniqlangan so'zlar ro'yxati shakllantiriladi; ma'lumotlar bazasining 1-ustuniga shu so'zlar kiritiladi. Keyingi ustunlarga darajalanish o'sib borishiga qarab so'zlar kiritiladi.

Qidiruv natijasiga esa ma'lumotlar bazasidagi ustunlar emas, balki qator ma'lumoti chiqariladi. Bazada nechta so' $z$ bo'lsa, shuncha qator shakllanadi. Qidiruv oynasiga baza tarkibiga kirgan so 'zlar orasidan qaysi so' $z$ kiritilsa ham, o'sha so' $z$ darajalanish qatorida ko'rsatiladi. Masalan:

\begin{tabular}{|l|l|l|}
\hline $\begin{array}{l}\text { ajablanmo } \\
\text { q }\end{array}$ & $\begin{array}{l}\text { hayron } \\
\text { bo'lmoq }\end{array}$ & $\begin{array}{l}\text { hayratlan } \\
\text { moq }\end{array}$ \\
\hline $\begin{array}{l}\text { achchiqlan } \\
\text { moq }\end{array}$ & $\begin{array}{l}\text { g'azablan }^{\text {moq }} \\
\text { moq }\end{array}$ & $\begin{array}{l}\text { qahrlanm } \\
\text { oq }\end{array}$ \\
\hline ahmoq & tentak & devona \\
\hline
\end{tabular}

O'zbek tili partonimik munosabat ifodalovchi birliklar

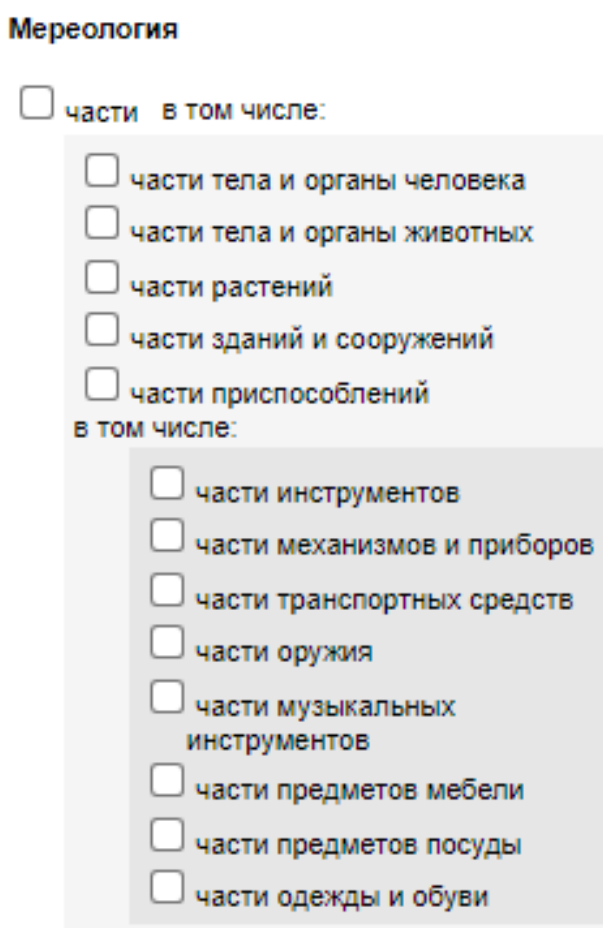

кванты и порции вещества множества и совокупности объектов имена классов 
bazasi. Til - ijtimoiy ongning shakli, ko'rinishi. Lisoniy partonimik munosabatlar borliqdagi butun-bo'lak munosabatlarning tildagi in'ikosidir [[Qilichev, 2002: 5son; Qilichev, 2002: 6-son; Qilichev, 1996]. Butunlik ma'lum tashkil etuvchilar bo'laklarning dialektik birlashmasi, unda har bir a'zo - bo'lak aniq bir funksiyani bajaradi. Bo'laklarning shunchaki to'dasi butunlikni yuzaga keltirmaydi. Masalan, barg, tana, yaproq, meva - bu hali daraxt degani emas. Butunlikning eng kichik bo'laklarigacha ma'lum so'z bilan ifodalanadi. Partonimik munosabat - shu butun va bo' laklarni atovchi so'zlararo aloqadorlik. Partonimiya hodisasida ikki atamani farqlash lozim. Bular: butunnom va bo'laknom. Butunnom - butunni atovchi so'z, u bo 'laknomlarni o'z ichiga qamrab oladi: "bosh" - tepa, peshana, ensa, yuz qatori uchun butunnom hisoblanadi. Bo'laknom - bo'lakni ifodalovchi so'z: yelka, ko'krak, qorin, bel kabilar tana uchun bo'laknom sanaladi. O'zbek tilida butunbo'lak munosabatni aks ettiruvchi ma' lumotlar bazasini shakllantirish zarur. Zotan, dunyoga mashhur til korpuslarida so'zlarning qismlarini bildiruvchi so'zlarni avtomatik qidirish imkoni mavjud. Masalan, rus tili milliy korpusining semantik belgilar asosidagi qidiruv qismida "Mereologiya" qismi qismni atovchi birliklar qidiruviga mo'ljallangan (1-rasm).

1 -rasm

$\mathrm{O}^{`}$ zbek tilida bunday bazani shakllantirish uchun ot turkumiga mansub so'zlar bazasi ajratib olinib, butun/qismni bildiruvchi so'z ekanligi haqida axborot kiritilishi lozim. Hatto bazani ajratmay, morfoleksikonning o'ziga bitta ustun qo'shish kifoya qiladi. O'zbek tili ta'limiy korpusida partonimik munosabat ifodalovchi birliklar bazasiga oid jadval shakllarini quyidagicha tavsiya qilish mumkin:

\begin{tabular}{|c|c|c|c|c|c|c|c|c|c|c|c|c|c|c|c|}
\hline $\mathbf{t} / \mathbf{r}$ & $\tilde{\phi}^{N}$ & 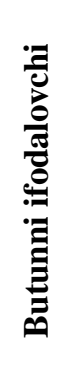 & 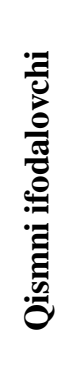 & 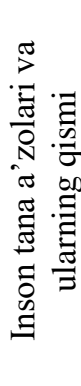 & 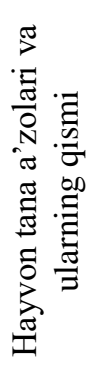 & 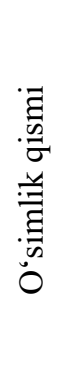 & 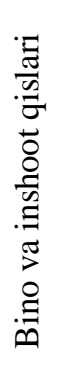 & 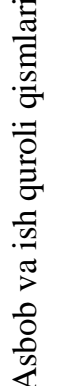 & 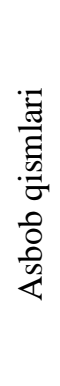 & 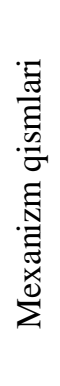 & 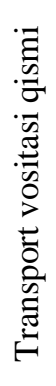 & $\begin{array}{l}\overrightarrow{\tilde{E}} \\
\vec{a} \\
\bar{\sigma} \\
\stackrel{0}{0} \\
0\end{array}$ & 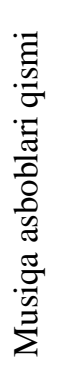 & 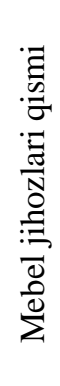 & 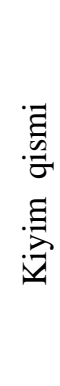 \\
\hline & & & & & & & & & & & & & & & \\
\hline
\end{tabular}

Umuman olganda, korpusdan foydalanuvchi qidirayotgan so' $z$ haqida batafsil ma'lumotni bir joydan topishiga erishish uchun so'zlararo ma'no munosabatlarini barchasining bazasini yaratish lozim. Sinonim, antonim birliklar 
bazasi uchun yetarli manbalar, lug'atlar mavjud. Lekin giponim/giperonim, xolo/meronimlar bo 'yicha tadqiqotlar mavjud bo'lsa-da, lug'atlar yaratilmagan. Korpus uchun giponim/giperonim, xolo/meronimlar bazasini yuqoridagi tavsiyalar bo'yicha ishlab chiqish mumkin.

\section{Foydalanilgan adabiyotlar:}

1. Ахмедова Д.Б. Атов бирликларини ўзбек тили корпуслари учун лексик-семантик теглашнинг лингвистик асос ва моделлари: филология фанлари бўйича фалсафа доктори (PhD) диссертацияси. - Бухоро, 2020. - 138 б.

2. Захаров В.П., Богданова С.Ю. Корпусная лингвистика. Учебник для студентов гуманитарных вузов. - Иркутск: ИГЛУ, 2011. - 161 с.

3. Лайонз Дж. Введение в теоретическую лингвистику. Москва, 1978.

4. Орифжонова Ш. Ўзбек тилида луғавий градуонимия: филол. фан. номз. дисс. автореф. - Тошкент, 1996. - 21 б.

5. Сафарова Р. Ўзбек тилида гипонимия: филол. фан. номз. диссер. автореф. - Тошкент., 1987. - 26 б.

6. Хамроева Ш. Ўзбек тили муаллифлик корпусини тузишнинг лингвистик асослари: Филол.фан.бўйича фалсафа доктори $(\mathrm{PhD})$...дис. Бухоро, 2018. - 165 б.

7. Эшмўминов А.А. Ўзбек тили миллий корпуси синоним сўзлар базасини яратиш асослари: филология фанлари буйича фалсафа доктори (PhD) диссертацияси. - Термиз, 2019. - 146 б.

8. Қиличев Б. Партонимиянинг синонимия, гипонимия ва градуонимияга муносабати // «Ўзбек тили ва адабиёти», 2002, 5-сон.

9. Қиличев Б. Тил ва нутқни фарқлаш хусусида // «Тил ва адабиёт таълими» журнали, 2002, 6-сон.

10. Қиличев Б. Ўзбек тилида партонимия ходисаси // «Тил ва адабиёт таълими» журнали, 1996, 5-сон.

11. Қиличев Б. Ўзбек тилида партонимия: филол. фан. номз. дисс. автореф. - Тошкент, 1996. - 25 б. 